This postprint is published in:

Journal of differential equations, Volumen 267, Issue 11, November 2019, 6539-6554

DOI: 10.1016/j.jde.2019.07.018

\title{
POSITIVE SOLUTIONS FOR A CLASS OF SINGULAR DIRICHLET PROBLEMS
}

\author{
N.S. PAPAGEORGIOU, V.D. RĂDULESCU, AND D.D. REPOVŠ
}

\begin{abstract}
We consider a Dirichlet elliptic problem driven by the Laplacian with singular and superlinear nonlinearities. The singular term appears on the left-hand side while the superlinear perturbation is parametric with parameter $\lambda>0$ and it need not satisfy the AR-condition. Having as our starting point the work of Diaz-Morel-Oswald (1987), we show that there is a critical parameter value $\lambda_{*}$ such that for all $\lambda>\lambda_{*}$ the problem has two positive solutions, while for $\lambda<\lambda_{*}$ there are no positive solutions. What happens in the critical case $\lambda=\lambda_{*}$ is an interesting open problem.
\end{abstract}

\section{INTRODUCTION}

Let $\Omega \subseteq \mathbb{R}^{N}(\mathbb{N} \geqslant 2)$ be a bounded domain with a $C^{2}$-boundary $\partial \Omega$. In this paper we study the following parametric singular Dirichlet problem

$\left(P_{\lambda}\right) \quad-\Delta u(z)+u(z)^{-\gamma}=\lambda f(z, u(z))$ in $\Omega,\left.u\right|_{\partial \Omega}=0, u>0,0<\gamma<1$.

In this problem, $\lambda$ is a positive parameter and $f: \Omega \times \mathbb{R} \rightarrow \mathbb{R}$ is a Carathéodory function (that is, for all $x \in \mathbb{R}$, the mapping $z \mapsto f(z, x)$ is measurable and for almost all $z \in \Omega$, the mapping $x \mapsto f(z, x)$ is continuous). We assume that for almost all $z \in \Omega, f(z, \cdot)$ exhibits superlinear growth near $+\infty$, but it need not satisfy the usual in such cases Ambrosetti-Robinowitz condition (the AR-condition for short).

The distinguishing feature of our work is that the singular term $u^{-\gamma}$ appears on the left-hand side of the equation. This is in contrast with almost all previous works on singular elliptic equations driven by the Laplacian, where the forcing term (the right-hand side of the equation) is $u \mapsto u^{-\gamma}+\lambda f(z, u)$, so the singular term $u^{-\gamma}$ appears on the right-hand side of the equation. We mention the works of Coclite \& Palmieri [2], Sun, Wu \& Long [13], and Haitao [7], which also deal with equations that have the competing effects of singular and superlinear terms. A comprehensive bibliography on semilinear singular Dirichlet problems can be found in the book by Ghergu \& Rădulescu [5]. The present class of singular equations was first considered by Diaz, Morel \& Oswald [3], for the case when the perturbation $f$ is independent of $u$. They produced a necessary and sufficient condition for the existence of positive solutions in terms of the integral $\int_{\Omega} f \hat{u}_{1} d z$, with $\hat{u}_{1}$ being the positive $L^{2}$-normalized principal eigenfunction of $\left(-\Delta, H_{0}^{1}(\Omega)\right)$. More recently, Papageorgiou \& Rădulescu [10] considered problem $\left(P_{\lambda}\right)$ with $f(z, \cdot)$ being sublinear.

Key words and phrases. Singular term, superlinear perturbation, weak comparison, order cone. 2010 Mathematics Subject Classification: 35J20, 35J60, 35J75. 
Our aim is to study the precise dependence of the set of positive solutions of problem $\left(P_{\lambda}\right)$ with respect to the parameter $\lambda>0$. In this direction, we show that there exists a critical parameter value $\lambda_{*}>0$ such that

- for all $\lambda>\lambda_{*}$, problem $\left(P_{\lambda}\right)$ has at least two positive smooth solutions;

- for all $\lambda \in\left(0, \lambda_{*}\right)$, problem $\left(P_{\lambda}\right)$ has no positive solutions.

It is an open problem what happens in the critical case $\lambda=\lambda_{*}$. We describe the difficulties one encounters when treating the critical case $\lambda=\lambda_{*}$ and why we think that $\lambda_{*}>0$ is not admissible.

\section{Preliminaries And hypotheses}

Let $X$ be a Banach space and $\varphi \in C^{1}(X, \mathbb{R})$. We say that $\varphi(\cdot)$ satisfies the "C-condition", if the following property holds

"Every sequence $\left\{u_{n}\right\}_{n \geqslant 1} \subseteq X$ such that

$\left\{\varphi\left(u_{n}\right)\right\}_{n \geqslant 1} \subseteq \mathbb{R}$ is bounded and $\left(1+\left\|u_{n}\right\|_{X}\right) \varphi^{\prime}\left(u_{n}\right) \rightarrow 0$ in $X^{*}$ as $n \rightarrow \infty$, admits a strongly convergent subsequence".

This is a compactness-type condition on the functional $\varphi$ and it leads to the minimax theory of the critical values of $\varphi$ (see, for example, Papageorgiou, Rădulescu \& Repovš [12]).

The main spaces used in the analysis of problem $\left(P_{\lambda}\right)$ are the Sobolev space $H_{0}^{1}(\Omega)$ and the Banach space $C_{0}^{1}(\bar{\Omega})=\left\{u \in C^{1}(\bar{\Omega}):\left.u\right|_{\partial \Omega}=0\right\}$. By $\|\cdot\|$ we denote the norm of $H_{0}^{1}(\Omega)$. On account of the Poincaré inequality we have

$$
\|u\|=\|D u\|_{2} \text { for all } u \in H_{0}^{1}(\Omega) .
$$

The Banach space $C_{0}^{1}(\bar{\Omega})$ is an ordered Banach space with positive cone

$$
C_{+}=\left\{u \in C_{0}^{1}(\bar{\Omega}): u(z) \geqslant 0 \text { for all } z \in \bar{\Omega}\right\} .
$$

This cone has a nonempty interior given by

$$
\operatorname{int} C_{+}=\left\{u \in C_{+}: u(z)>0 \text { for all } z \in \Omega,\left.\frac{\partial u}{\partial n}\right|_{\partial \Omega}<0\right\},
$$

with $n(\cdot)$ being the outward unit normal on $\partial \Omega$.

We will also use two other ordered Banach spaces, namely $C^{1}(\bar{\Omega})$ and

$$
C_{0}(\bar{\Omega})=\left\{u \in C(\bar{\Omega}):\left.u\right|_{\partial \Omega}=0\right\} .
$$

The order cones are

$$
\hat{C}_{+}=\left\{u \in C^{1}(\bar{\Omega}): u(z) \geqslant 0 \text { for all } z \in \bar{\Omega}\right\}
$$

and

$$
K_{+}=\left\{u \in C_{0}(\bar{\Omega}): u(z) \geqslant 0 \text { for all } z \in \bar{\Omega}\right\},
$$

respectively. Both have nonempty interiors given by

$$
\begin{aligned}
& D_{+}=\left\{u \in \hat{C}_{+}: u(z)>0 \text { for all } z \in \bar{\Omega}\right\}, \\
& \check{K}_{+}=\left\{u \in K_{+}: c_{u} \hat{d} \leqslant u \text { for some } c_{u}>0\right\},
\end{aligned}
$$

with $\hat{d}(z)=d(z, \partial \Omega)$ for all $z \in \bar{\Omega}$.

Concerning ordered Banach spaces, the following result is helpful (see Papageorgiou, Rădulescu \& Repovš [12, Proposition 4.1.22, p. 226]). 
Proposition 1. If $X$ is an ordered Banach space with order (positive) cone $K$, int $K \neq \emptyset$, and $e \in \operatorname{int} K$, then for every $u \in X$, we can find $\lambda_{u}>0$ such that $\lambda_{u} e-u \in K$.

Next, we introduce the main notation which we will use in the sequel. Given $\varphi \in C^{1}\left(H_{0}^{1}(\Omega)\right)$, we denote by $K_{\varphi}$ the critical set of $\varphi$, that is,

$$
K_{\varphi}=\left\{u \in H_{0}^{1}(\Omega): \varphi^{\prime}(u)=0\right\} .
$$

For $x \in \mathbb{R}$, we set $x^{ \pm}=\max \{ \pm x, 0\}$. Given $u \in H_{0}^{1}(\Omega)$, we set $u^{ \pm}(z)=u(z)^{ \pm}$ for all $z \in \Omega$. We know that

$$
u^{ \pm} \in H_{0}^{1}(\Omega), u=u^{+}-u^{-},|u|=u^{+}+u^{-} .
$$

Given $u, y \in H_{0}^{1}(\Omega)$ with $u \leqslant y$, we define

$$
\begin{aligned}
& {[u, y]=\left\{h \in H_{0}^{1}(\Omega): u(z) \leqslant h(z) \leqslant y(z) \text { for almost all } z \in \Omega\right\},} \\
& {[u)=\left\{h \in H_{0}^{1}(\Omega): u(z) \leqslant h(z) \text { for almost all } z \in \Omega\right\} .}
\end{aligned}
$$

Also, by

$$
\operatorname{int}_{C_{0}^{1}(\bar{\Omega})}[u, y]
$$

we denote the interior of $[u, y] \cap C_{0}^{1}(\bar{\Omega})$ in the $C_{0}^{1}(\bar{\Omega})$-norm topology.

By $\hat{\lambda}_{1}>0$ we denote the principal eigenvalue of $\left(-\Delta, H_{0}^{1}(\Omega)\right)$ and by $\hat{u}_{1}$ the corresponding positive $L^{2}$-normalized (that is, $\left\|\hat{u}_{1}\right\|_{2}=1$ ) eigenfunction. Standard regularity theory and the Hopf maximum principle imply that $\hat{u}_{1} \in \operatorname{int} C_{+}$.

Finally, by $2^{*}$ we denote the critical Sobolev exponent, $2^{*}=\left\{\begin{array}{ll}\frac{2 N}{N-2} & \text { if } N \geqslant 3 \\ +\infty & \text { if } N=2\end{array}\right.$.

Now we will introduce our hypotheses on the perturbation $f(z, x)$.

$H(f): f: \Omega \times \mathbb{R} \rightarrow \mathbb{R}$ is a Carathéodory function such that $f(z, 0)=0$ for almost all $z \in \Omega$ and

(i) $f(z, x) \leqslant a(z)\left(1+x^{r-1}\right)$ for almost all $z \in \Omega$ and all $x \geqslant 0$, with $a \in L^{\infty}(\Omega)$, $2<r<2^{*}$

(ii) if $F(z, x)=\int_{0}^{x} f(z, s) d s$, then $\lim _{x \rightarrow+\infty} \frac{F(z, x)}{x^{2}}=+\infty$ uniformly for almost all $z \in \Omega$;

(iii) there exists $\tau \in\left(r-2,2^{*}\right)$ such that $0<\beta_{0} \leqslant \liminf _{x \rightarrow \infty} \frac{f(z, x) x-2 F(z, x)}{x^{\tau}}$ uniformly for almost all $z \in \Omega ;$

(iv) for every $\rho>0$ and every $\lambda>0$, there exists $\hat{\xi}_{\rho}^{\lambda}>0$ such that for almost all $z \in \Omega$, the function

$$
x \mapsto \lambda f(z, x)+\hat{\xi}_{\rho}^{\lambda} x
$$

is nondecreasing on $[0, \rho]$ and for every $s>0$ we have

$$
\inf \{f(z, x): x \geqslant s\}=m_{s}>0 \text { for almost all } z \in \Omega ;
$$

(v) there exist $q>2, \delta_{0}>0, \hat{c}>0$ such that

$$
\begin{aligned}
& \hat{c} x^{q-1} \leqslant f(z, x) \text { for almost all } z \in \Omega \text { and all } 0 \leqslant x \leqslant \delta_{0}, \\
& \lim _{x \rightarrow 0^{+}} \frac{F(z, x)}{x^{2}}=0 \text { uniformly for almost all } z \in \Omega .
\end{aligned}
$$


Remark 1. Since we are looking for positive solutions and all of the above hypotheses concern the positive semiaxis $\mathbb{R}_{+}=[0,+\infty)$, we may assume without any loss of generality that

$$
f(z, x)=0 \text { for almost all } z \in \Omega \text { and all } x \leqslant 0 .
$$

Hypotheses H(f)(ii),(iii) imply that

$$
\lim _{x \rightarrow+\infty} \frac{f(z, x)}{x}=+\infty \text { uniformly for almost all } z \in \Omega .
$$

So, the perturbation $f(z, \cdot)$ is superlinear. However, we do not express this superlinearity of $f(z, \cdot)$ by using the traditional (for superlinear problems) AR-condition. We recall that the AR-condition (the unilateral version due to (1)) says that there exist $\vartheta>2$ and $M>0$ such that

$$
\begin{aligned}
& 0<\vartheta F(z, x) \leqslant f(z, x) x \text { for almost all } z \in \Omega \text { and all } x \geqslant M \\
& 0<\underset{\Omega}{\operatorname{essinf}} F(\cdot, M) .
\end{aligned}
$$

Integrating (2a) and using (2b), we obtain the following weaker condition

$$
\begin{aligned}
& c_{1} x^{\vartheta} \leqslant F(z, x) \text { for almost all } z \in \Omega, \text { all } x \geqslant M, \text { and some } c_{1}>0, \\
\Rightarrow \quad & c_{1} x^{\vartheta-1} \leqslant f(z, x) \text { for almost all } z \in \Omega \text { and all } x \geqslant M \text { (see (2a)). }
\end{aligned}
$$

So, the AR-condition dictates at least $(\vartheta-1)$-polynomial growth for $f(z, \cdot)$. Here, instead of the AR-condition, we employ hypothesis $H(f)(i i i)$ which is less restrictive and incorporates in our framework superlinear nonlinearities with "slower" growth near $+\infty$. Consider the following function (for the sake of simplicity we drop the z-dependence)

$$
f(x)= \begin{cases}c x^{q-1} & \text { if } 0 \leqslant x \leqslant 1 \\ x \ln x+c x^{\vartheta-1} & \text { if } 1<x\end{cases}
$$

with $c>0, q>2>\vartheta>1$ (see (1)). Then $f(\cdot)$ satisfies hypotheses $H(f)$ but it fails to satisfy the AR-condition.

Finally, we mention that for $u \in H_{0}^{1}(\Omega)$ we have

$$
c_{u} \hat{d} \leqslant u \text { for some } c_{u}>0 \text { if and only if } \hat{c}_{u} \hat{u}_{1} \leqslant u \text { for some } \hat{c}_{u}>0 \text {. }
$$

For $\delta>0$ let $\Omega_{\delta}=\{z \in \Omega: d(z, \partial \Omega)<\delta\}$ and let $\tilde{C}^{1}\left(\Omega_{\delta}\right)=\left\{u \in C^{1}\left(\bar{\Omega}_{\delta}\right):\right.$ $\left.\left.u\right|_{\partial \Omega}=0\right\}$ with order cone

$$
\tilde{C}^{1}\left(\bar{\Omega}_{\delta}\right)_{+}=\left\{u \in \tilde{C}^{1}\left(\bar{\Omega}_{\delta}\right): u(z) \geqslant 0 \text { for all } z \in \bar{\Omega}_{\delta}\right\},
$$

which has nonempty interior given by

$$
\operatorname{int} \tilde{C}^{1}\left(\bar{\Omega}_{\delta}\right)_{+}=\left\{u \in \tilde{C}^{1}\left(\bar{\Omega}_{\delta}\right)_{+}: u(z)>0 \text { for all } z \in \Omega_{\delta} \text { and }\left.\frac{\partial u}{\partial n}\right|_{\partial \Omega}<0\right\} .
$$

According to Lemma 14.16 of Gilbarg \& Trudinger [6, p. 355], for $\delta>0$ small enough we have $\hat{d} \in \operatorname{int} \tilde{C}^{1}\left(\bar{\Omega}_{\delta}\right)_{+}$. Also, we have $\hat{d} \in D_{+}\left(\bar{\Omega} \backslash \Omega_{\delta}\right)$, with the latter being the interior of the order cone of $C^{1}\left(\bar{\Omega} \backslash \Omega_{\delta}\right)$. So, using Proposition 1 we can find $0<\hat{c}_{1}<\hat{c}_{2}$ such that $\hat{c_{1}} \hat{d} \leqslant \hat{u}_{1} \leqslant \hat{c}_{2} \hat{d}$ (recall that $\hat{u}_{1} \in \operatorname{int} C_{+}$). This implies (3). 


\section{Positive solutions}

Let $\eta>0$. We start by considering the following auxiliary purely singular Dirichlet problem

$(A u)_{\eta} \quad-\Delta u(z)+u(z)^{-\gamma}=\eta \hat{u}_{1}(z)$ in $\Omega,\left.u\right|_{\partial \Omega}=0$.

By Theorem 1 of Diaz, Morel \& Oswald [3] we know that for $\eta>0$ big problem $(A u)_{\eta}$ has a solution $v_{\eta} \in H_{0}^{1}(\Omega) \cap C_{0}(\bar{\Omega})$ and $v_{\eta}^{-\gamma} \in L^{1}(\Omega), c_{\eta} \hat{u}_{1} \leqslant v_{\eta}$ for some $c_{\eta}>0$.

Also, we consider the following Dirichlet problem

$$
-\Delta u(z)=\lambda f(z, u(z)) \text { in } \Omega,\left.u\right|_{\partial \Omega}, u>0, \lambda>0 .
$$

Proposition 2. If hypotheses $H(f)$ hold and $\lambda>0$, then problem $\left(Q_{\lambda}\right)$ has a solution $\hat{u}_{\lambda} \in \operatorname{int} C_{+}$.

Proof. Let $\Psi_{\lambda}: H_{0}^{1}(\Omega) \rightarrow \mathbb{R}$ be the $C^{1}$ - functional defined by

$$
\Psi_{\lambda}(u)=\frac{1}{2}\|D u\|_{2}^{2}-\int_{\Omega} \lambda F(z, u) d z \text { for all } u \in H_{0}^{1}(\Omega) .
$$

Hypotheses $H(f)($ ii $),($ iii) imply that

$$
\Psi_{\lambda}(\cdot) \text { satisfies the C-condition }
$$

(see Papageorgiou \& Rădulescu [11, Proposition 9]).

Combining hypotheses $H(f)(i),(v)$, given $\epsilon>0$, we can find $c_{\epsilon}>0$ such that

$$
F(z, x) \leqslant \frac{\epsilon}{2} x^{2}+c_{\epsilon} x^{r} \text { for almost all } z \in \Omega, \text { all } x \geqslant 0 .
$$

$$
\begin{aligned}
\Psi_{\lambda}(u) & \geqslant \frac{1}{2}\|D u\|_{2}^{2}-\frac{\lambda \epsilon}{2}\|u\|_{2}^{2}-\lambda \hat{c}_{\epsilon}\|u\|^{r} \text { for some } \hat{c}_{\epsilon}>0 \\
& \geqslant c_{2}\|u\|^{2}-\lambda \hat{c}_{\epsilon}\|u\|^{r} \text { for some } c_{2}=c_{2}(\lambda)>0 \text { (choose } \epsilon>0 \text { small enough), } \\
& \Rightarrow u=0 \text { is a local minimizer of } \Psi_{\lambda}(\cdot)(\text { recall that } r>2) .
\end{aligned}
$$

We can easily see that if $u \in K_{\Psi_{\lambda}}$, then $u \geqslant 0$. Hence we assume that $K_{\Psi_{\lambda}}$ is finite. On account of (5) and Theorem 5.7.6 of Papageorgiou, Rădulescu \& Repovš $[12$, p. 367], we can find $\rho \in(0,1)$ so small that

$$
0=\Psi_{\lambda}(0)<\inf \left\{\Psi_{\lambda}(u):\|u\|=\rho\right\}=m_{\lambda} .
$$

Hypothesis $H(f)(i i)$ implies that

$$
\Psi_{\lambda}\left(t \hat{u}_{1}\right) \rightarrow-\infty \text { as } t \rightarrow+\infty .
$$

Then (4), (6), (7) permit the use of the mountain pass theorem. So, we can find $\hat{u}_{\lambda} \in H_{0}^{1}(\Omega)$ such that

$$
\begin{aligned}
& \hat{u}_{\lambda} \in K_{\Psi_{\lambda}} \text { and } m_{\lambda} \leqslant \Psi_{\lambda}\left(\hat{u}_{\lambda}\right), \\
& \Rightarrow \hat{u}_{\lambda} \geqslant 0, \hat{u}_{\lambda} \neq 0(\operatorname{see}(6)) .
\end{aligned}
$$

We have

$$
\begin{aligned}
& \int_{\Omega}\left(D \hat{u}_{\lambda}, D h\right)_{\mathbb{R}^{N}} d z=\lambda \int_{\Omega} f\left(z, u_{\lambda}\right) h d z \text { for all } h \in H_{0}^{1}(\Omega), \\
\Rightarrow & -\Delta u_{\lambda}(z)=\lambda f\left(z, u_{\lambda}(z)\right) \geqslant 0 \text { for almost all } z \in \Omega .
\end{aligned}
$$

Then the semilinear regularity theory (see Gilbarg \& Trudinger [6]) and the Hopf maximum principle (see Gasinski \& Papageorgiou [4]), imply that $\hat{u}_{\lambda} \in \operatorname{int} C_{+}$. 
Hypotheses $H(f)$ imply that we can find $c_{2}>0$ such that

$$
f(z, x) \geqslant c_{2} \min \left\{x, x^{q-1}\right\} \text { for almost all } z \in \Omega \text { and all } x \geqslant 0 .
$$

We have $\hat{u}_{\lambda} \in \operatorname{int} C_{+}$and $\hat{u}_{\lambda}^{q-1} \in \operatorname{int} K_{+}$. So, we can find $c_{3}>0$ such that

$$
\begin{aligned}
\eta \hat{u}_{1} & \leqslant c_{3} \hat{u}_{\lambda} \text { and } \eta \hat{u}_{1} \leqslant c_{3} \hat{u}_{\lambda}^{q-1}, \\
\Rightarrow \quad \eta \hat{u}_{1} & \leqslant c_{3} \min \left\{\hat{u}_{\lambda}, \hat{u}_{\lambda}^{q-1}\right\} .
\end{aligned}
$$

From (8) and (9) we see that we can find $\lambda_{0} \geqslant 0$ big such that for $\lambda \geqslant \lambda_{0}$

$$
\begin{aligned}
& \lambda f\left(z, \hat{u}_{\lambda}(z)\right) \geqslant \lambda_{0} c_{2} \min \left\{\hat{u}_{\lambda}(z), \hat{u}_{\lambda}(z)^{q-1}\right\} \\
& \geqslant \eta \hat{u}_{1}(z) \text { for almost all } z \in \Omega .
\end{aligned}
$$

Recall that $c_{\eta} \hat{u}_{1} \leqslant v_{\eta}$ for some $c_{\eta}>0$. Hence by $(3), \hat{c}_{\eta} \hat{d} \leqslant v_{\eta}$ for some $\hat{c}_{\eta}>0$. Therefore

$$
v_{\eta}^{-\gamma} \leqslant \frac{1}{\hat{c}_{\eta}^{\gamma}} \hat{d}^{-\gamma}
$$

For every $h \in H_{0}^{1}(\Omega)$, we have

$$
\begin{aligned}
& \int_{\Omega} \frac{|h|}{v_{\eta}^{\gamma}} d z \leqslant \frac{1}{\hat{c}_{\eta}^{\gamma}} \int_{\Omega} \frac{|h|}{\hat{d}^{\gamma}} d z \\
& =\frac{1}{\hat{c}_{\eta}^{\gamma}} \int_{\Omega} \frac{|h|}{\hat{d}} \hat{d}^{1-\gamma} d z \leqslant c_{4} \int_{\Omega} \frac{|h|}{\hat{d}} d z \text { for some } c_{4}>0 .
\end{aligned}
$$

Invoking Hardy's inequality (see Brezis [1, p. 313]), we infer that $\frac{|h|}{\hat{d}} \in L^{2}(\Omega)$. Therefore

$$
\begin{aligned}
& c_{4} \int_{\Omega} \frac{|h|}{\hat{d}} d z \leqslant c_{5}\left(\int_{\Omega} \frac{|h|^{2}}{\hat{d}^{2}} d z\right)^{\frac{1}{2}}<\infty \text { for some } c_{5}>0 \\
\Rightarrow \quad & \left|\int_{\Omega} v_{\eta}^{-\gamma} h d z\right|<\infty \text { for all } h \in H_{0}^{1}(\Omega) .
\end{aligned}
$$

Therefore we have

$$
-\Delta v_{\eta}(z)+v_{\eta}(z)^{-\gamma}=\eta \hat{u}_{1}(z) \text { for almost all } z \in \Omega .
$$

If $\lambda \geqslant \lambda_{0}$, from (10) and (11) we have

$$
\begin{array}{r}
-\Delta \hat{u}_{\lambda}(z)=\lambda f\left(z, \hat{u}_{\lambda}(z)\right) \geqslant \eta \hat{u}_{1}(z)=-\Delta v_{\eta}(z)+v_{\eta}(z)^{-\gamma} \geqslant-\Delta v_{\eta}(z) \\
\text { for almost all } z \in \Omega .
\end{array}
$$

Since $\left.v_{\eta}\right|_{\partial \Omega}=\left.\hat{u}_{\lambda}\right|_{\partial \Omega}=0$, from (12) and the weak comparison principle (see Tolksdorf [14, Lemma 3.1]), we have

$$
v_{\eta} \leqslant \hat{u}_{\lambda} \quad\left(\lambda \geqslant \lambda_{0}\right)
$$

Now we introduce the following two sets

$$
\begin{aligned}
& \mathcal{L}=\left\{\lambda>0: \text { problem }\left(P_{\lambda}\right) \text { has a positive solution }\right\} \\
& S_{\lambda}=\text { the set of positive solutions of }\left(P_{\lambda}\right) .
\end{aligned}
$$

Here by a solution of $\left(P_{\lambda}\right)$, following [10], we understand a function $u \in H_{0}^{1}(\Omega)$ such that

(a) $u \in L^{\infty}(\Omega), u(z)>0$ for almost all $z \in \Omega$, and $u^{-\gamma} \in L^{1}(\Omega)$;

(b) there exists $c_{u}>0$ such that $c_{u} \hat{d} \leqslant u$;

(c) $\int_{\Omega}(D u, D h)_{\mathbb{R}^{N}} d z+\int_{\Omega} u^{-\gamma} h d z=\lambda \int_{\Omega} f(z, u) h d z$ for all $h \in H_{0}^{1}(\Omega)$. 
From (3) we know that (b) is equivalent to saying that $\hat{c}_{u} \hat{u}_{1} \leqslant u$ for some $\hat{c}_{u}>0$. Also the previous discussion reveals that (c) makes sense. Regularity theory will provide additional structure for the solutions of $\left(P_{\lambda}\right)$.

Proposition 3. If hypotheses $H(f)$ hold, then $\mathcal{L} \neq \emptyset$ and $S(\lambda) \subseteq \operatorname{int} C_{+}$.

Proof. Let $\lambda \geqslant \lambda_{0}$. Using (13) we can introduce the Carathéodory function $g_{\lambda}(z, x)$ defined by

$$
g_{\lambda}(z, x)= \begin{cases}\lambda f\left(z, v_{\eta}(z)\right)-v_{\eta}(z)^{-\gamma} & \text { if } x<v_{\eta}(z) \\ \lambda f(z, x)-x^{-\gamma} & \text { if } v_{\eta}(z) \leqslant x \leqslant \hat{u}_{\lambda}(z) \\ \lambda f\left(z, \hat{u}_{\lambda}(z)\right)-\hat{u}_{\lambda}(z)^{-\gamma} & \text { if } \hat{u}_{\lambda}(z)<x .\end{cases}
$$

We set $G_{\lambda}(z, x)=\int_{0}^{x} g_{\lambda}(z, s) d s$ and consider the functional $\varphi_{\lambda}: H_{0}^{1}(\Omega) \rightarrow \mathbb{R}$ defined by

$$
\varphi_{\lambda}(u)=\frac{1}{2}\|D u\|_{2}^{2}-\int_{\Omega} G_{\lambda}(z, u) d z \text { for all } u \in H_{0}^{1}(\Omega) .
$$

From Papageorgiou \& Rădulescu [10] (see Claim 1 in the proof of Proposition 6 ), we have that $\varphi_{\lambda} \in C^{1}\left(H_{0}^{1}(\Omega)\right)$. It is clear from (14) that $\varphi_{\lambda}(\cdot)$ is coercive. Also, it is sequentially weakly lower semicontinuous. Therefore we can find $u_{\lambda} \in H_{0}^{1}(\Omega)$ such that

$$
\begin{aligned}
& \varphi_{\lambda}\left(u_{\lambda}\right)=\inf \left\{\varphi_{\lambda}(u): u \in H_{0}^{1}(\Omega)\right\}, \\
\Rightarrow \quad & \varphi_{\lambda}^{\prime}\left(u_{\lambda}\right)=0, \\
\Rightarrow & \int_{\Omega}^{1}\left(D u_{\lambda}, D h\right)_{\mathbb{R}^{N}} d z=\int_{\Omega} g_{\lambda}\left(z, u_{\lambda}\right) h d z \text { for all } h \in H_{0}^{1}(\Omega) .
\end{aligned}
$$

In (15) first we choose $h=\left(u_{\lambda}-\hat{u}_{\lambda}\right)^{+} \in H_{0}^{1}(\Omega)$. We have

$$
\begin{aligned}
\int_{\Omega}\left(D u_{\lambda}, D\left(u_{\lambda}-\hat{u}_{\lambda}\right)^{+}\right)_{\mathbb{R}^{N}} d z & =\int_{\Omega}\left[\lambda f\left(z, \hat{u}_{\lambda}\right)-\hat{u}_{\lambda}^{-\gamma}\right]\left(u_{\lambda}-\hat{u}_{\lambda}\right)^{+} d z(\text { see }(14)) \\
& \leqslant \int_{\Omega} \lambda f\left(z, \hat{u}_{\lambda}\right)\left(u_{\lambda}-\hat{u}_{\lambda}\right)^{+} d z \\
& =\int_{\Omega}\left(D \hat{u}_{\lambda}, D\left(u_{\lambda}-\hat{u}_{\lambda}\right)^{+}\right)_{\mathbb{R}^{N}} d z \text { (see Proposition 2), } \\
\Rightarrow\left\|D\left(u_{\lambda}-\hat{u}_{\lambda}\right)^{+}\right\|_{2}^{2} \leqslant 0, &
\end{aligned}
$$

Next, in (15) we choose $h=\left(v_{\eta}-u_{\lambda}\right)^{+} \in H_{0}^{1}(\Omega)$. Then we have

$$
\int_{\Omega}\left(D u_{\lambda}, D\left(v_{\eta}-u_{\lambda}\right)^{+}\right)_{\mathbb{R}^{N}} d z=\int_{\Omega}\left[\lambda f\left(z, v_{\eta}\right)-v_{\eta}^{-\gamma}\right]\left(v_{\eta}-u_{\lambda}\right)^{+} d z .
$$

As we proved (10), using (8), (9), we see that by taking $\lambda \geqslant \lambda_{0}$ even bigger if necessary, we can have

$$
\lambda f\left(z, v_{\eta}(z)\right) \geqslant \eta \hat{u}_{1}(z) \text { for almost all } z \in \Omega .
$$

Hence from (16) and (17) we have

$$
\begin{aligned}
\int_{\Omega}\left(D u_{\lambda}, D\left(v_{\eta}-u_{\lambda}\right)^{+}\right)_{\mathbb{R}^{N}} d z & \geqslant \int_{\Omega}\left[\eta \hat{u}_{1}-v_{\eta}^{-\gamma}\right]\left(v_{\eta}-u_{\lambda}\right)^{+} d z \\
& =\int_{\Omega}\left(D v_{\eta}, D\left(v_{\eta}-u_{\lambda}\right)^{+}\right)_{\mathbb{R}^{N}} d z \\
\Rightarrow\left\|D\left(v_{\eta}-u_{\lambda}\right)^{+}\right\|_{2}^{2} \leqslant 0, & \\
\Rightarrow v_{\eta} \leqslant u_{\lambda} . &
\end{aligned}
$$


So, we have proved that

$$
u_{\lambda} \in\left[v_{\eta}, \hat{u}_{\lambda}\right]
$$

It follows from (14), (15) and (18) that

$$
\int_{\Omega}\left(D u_{\lambda}, D h\right)_{\mathbb{R}^{N}} d z+\int_{\Omega} u_{\lambda}^{-\gamma} h d z=\int_{\Omega} f\left(z, u_{\lambda}\right) h d z \text { for all } h \in H_{0}^{1}(\Omega) .
$$

Recall that

$$
\begin{array}{ll}
c_{\eta} \hat{d} \leqslant v_{\eta} \leqslant u_{\lambda} \\
\text { and } \quad & u_{\lambda}^{-\gamma} \leqslant v_{\eta}^{-\gamma} \in L^{1}(\Omega)(\text { see }(18)) .
\end{array}
$$

Therefore $u_{\lambda}$ is a solution of $\left(P_{\lambda}\right)$. We have proved that for $\lambda \geqslant \lambda_{0}$ big enough, we have $\lambda \in \mathcal{L}$ and so $\mathcal{L} \neq \emptyset$.

Now let $u \in S_{\lambda}$. Then by definition we have

$$
\begin{aligned}
& \hat{c}_{u} \hat{u}_{1} \leqslant u \text { for some } \hat{c}_{u}>0, \\
\Rightarrow \quad & u \in \operatorname{int} K_{+} .
\end{aligned}
$$

Let $s>N$. Since $\hat{u}_{1}^{1 / s} \in K_{+}$, we can find $c_{6}>0$ such that

$$
\begin{aligned}
& \hat{u}_{1}^{1 / s} \leqslant c_{6} u(\text { see Proposition } 1), \\
\Rightarrow \quad & u^{-\gamma} \leqslant c_{7} \hat{u}_{1}^{-\frac{\gamma}{s}} \text { for some } c_{7}>0 .
\end{aligned}
$$

However, by Lemma in Lazer \& McKenna [8], we have that $\hat{u}_{1}^{-\frac{\gamma}{s}} \in L^{s}(\Omega)$ (recall that $0<\gamma<1)$. So, it follows that $u^{-\gamma} \in L^{s}(\Omega)$. Then Theorem 9.15 of Gilbarg \& Trudinger [6, p. 241] implies that $u \in W^{2, s}(\Omega)$. Since $s>N$, from the Sobolev embedding theorem, we have $u \in C^{1, \alpha}(\bar{\Omega})$ with $\alpha=1-\frac{N}{s}$. We conclude that $u \in \operatorname{int} C_{+}\left(\right.$see (19)) and so $S_{\lambda} \subseteq \operatorname{int} C_{+}$.

Next, we prove a structural property for the set $\mathcal{L}$ and a kind of monotonicity property for the set $S_{\lambda}$ with respect to $\lambda \in \mathcal{L}$.

Proposition 4. If hypotheses $H(f)$ hold, $\lambda \in \mathcal{L}, \mu>\lambda$, and $u_{\lambda} \in S_{\lambda} \subseteq \operatorname{int} C_{+}$, then $\mu \in \mathcal{L}$ and we can find $u_{\mu} \in S_{\mu} \subseteq \operatorname{int} C_{+}$.

Proof. Let $\rho=\left\|u_{\lambda}\right\|_{\infty}$. Hypotheses $H(f)$ imply that we can find $c_{\rho}>0$ such that

$$
0 \leqslant f(z, x) \leqslant c_{\rho} x \text { for almost all } z \in \Omega \text { and all } 0 \leqslant x \leqslant \rho .
$$

Also from (8) we know that

$$
f(z, x) \geqslant c_{2} \min \left\{x, x^{q-1}\right\} \text { for almost all } z \in \Omega \text { and all } x \geqslant 0 .
$$

Recall that for $\vartheta \geqslant \lambda_{0}$ we have $\hat{u}_{\theta} \geqslant v_{\eta}$ (see (13)) and $v_{\eta} \in \operatorname{int} K_{+}$. So, for $\vartheta \geqslant \lambda_{0}$ big enough we have

$$
\vartheta c_{2} \min \left\{\hat{u}_{\theta}, \hat{u}_{\theta}^{q-1}\right\} \geqslant \lambda c_{\rho} u_{\lambda}
$$

It follows that

$$
\begin{aligned}
-\Delta \hat{u}_{\theta}=\vartheta f\left(z, \hat{u}_{\theta}\right) & \geqslant \vartheta c_{2} \min \left\{\hat{u}_{\theta}, \hat{u}_{\theta}^{q-1}\right\}(\text { see }(21)) \\
& \geqslant \lambda c_{\rho} u_{\lambda}(\text { see }(22)) \\
& \geqslant \lambda f\left(z, u_{\lambda}\right)(\text { see }(20)) \\
& =-\Delta u_{\lambda}+u_{\lambda}^{-\gamma}\left(\text { since } u_{\lambda} \in S_{\lambda}\right) \\
& \geqslant-\Delta u_{\lambda} \text { for almost all } z \in \Omega,
\end{aligned}
$$

$\Rightarrow \hat{u}_{\theta} \geqslant u_{\lambda}$ (by the weak comparison principle, see Tolksdorf [14]). 
Therefore we can introduce the Carathéodory function $k_{\mu}(z, x)$ defined by

$$
k_{\mu}(z, x)= \begin{cases}\mu f\left(z, u_{\lambda}(z)\right)-u_{\lambda}(z)^{-\gamma} & \text { if } x<u_{\lambda}(z) \\ \mu f(z, x)-x^{-\gamma} & \text { if } u_{\lambda}(z) \leqslant x \leqslant \hat{u}_{\theta}(z) \\ \mu f\left(z, \hat{u}_{\theta}(z)\right)-\hat{u}_{\theta}(z)^{-\gamma} & \text { if } \hat{u}_{\theta}(z)<x\end{cases}
$$

We set $K_{\mu}(z, x)=\int_{0}^{x} k_{\mu}(z, s) d s$ and consider the functional $\sigma_{\mu}: H_{0}^{1}(\Omega) \rightarrow \mathbb{R}$ defined by

$$
\sigma_{\mu}(u)=\frac{1}{2}\|D u\|_{2}^{2}-\int_{\Omega} k_{\mu}(z, u) d z \text { for all } u \in H_{0}^{1}(\Omega) .
$$

Again we have $\sigma_{\mu} \in C^{1}\left(H_{0}^{1}(\Omega)\right.$ ) (see Papageorgiou \& Rădulescu [10]). From (23) it is clear that $\sigma_{\mu}(\cdot)$ is coercive. Also, by the Sobolev embedding theorem we see that $\sigma_{\mu}(\cdot)$ is sequentially weakly lower semicontinuous. So, by the WeierstrassTonelli theorem, we can find $u_{\mu} \in H_{0}^{1}(\Omega)$ such that

$$
\begin{aligned}
& \sigma_{\mu}\left(u_{\mu}\right)=\inf \left\{\sigma_{\mu}(u): u \in H_{0}^{1}(\Omega)\right\} \\
\Rightarrow & \sigma_{\mu}^{\prime}\left(u_{\mu}\right)=0, \\
\Rightarrow & \int_{\Omega}\left(D u_{\lambda}, D h\right)_{\mathbb{R}^{N}} d z=\int_{\Omega} k_{\mu}\left(z, u_{\lambda}\right) h d z \text { for all } h \in H_{0}^{1}(\Omega) .
\end{aligned}
$$

Choosing first $h=\left(u_{\mu}-\hat{u}_{\theta}\right)^{+} \in H_{0}^{1}(\Omega)$ and then $h=\left(u_{\lambda}-u_{\mu}\right)^{+} \in H_{0}^{1}(\Omega)$ as in the proof of Proposition 3, we can show that

$$
\begin{aligned}
& u_{\mu} \in\left[u_{\lambda}, \hat{u}_{\theta}\right], \\
\Rightarrow \quad & u_{\mu} \in S_{\mu} \subseteq \operatorname{int} C_{+}(\text {see }(23)) .
\end{aligned}
$$

Let $\rho=\left\|\hat{u}_{\theta}\right\|_{\infty}$ and let $\hat{\xi}_{0}=\max \left\{\hat{\xi}_{\rho}^{\lambda}, \hat{\xi}_{\rho}^{\mu}\right\}$ (see hypothesis $H(f)(i v)$ ). We have

$$
\begin{aligned}
&-\Delta u_{\lambda}+\hat{\xi}_{0} u_{\lambda}=\lambda f\left(z, u_{\lambda}\right)+\hat{\xi}_{0} u_{\lambda}-u_{\lambda}^{-\gamma} \\
& \leqslant \mu f\left(z, u_{\mu}\right)+\hat{\xi}_{0} u_{\mu}-u_{\mu}^{-\gamma} \\
&(\text { see hypothesis } H(f)(i v) \text { and }(24)) \\
&=-\Delta u_{\mu}+\hat{\xi}_{0} u_{\mu}\left(\text { since } u_{\mu} \in S_{\mu}\right) \\
& \Rightarrow \Delta\left(u_{\mu}-u_{\lambda}\right) \leqslant \hat{\xi}_{0}\left(u_{\mu}-u_{\lambda}\right), \\
& \Rightarrow u_{\mu}-u_{\lambda} \in \operatorname{int} C_{+} \text {(by Hopf's maximum principle). }
\end{aligned}
$$

The proof is now complete.

This proposition implies that $\mathcal{L}$ is a half-line. More precisely, let $\lambda_{*}=\inf \mathcal{L}$. We have

$$
\left(\lambda_{*},+\infty\right) \subseteq \mathcal{L} \subseteq\left[\lambda_{*},+\infty\right) .
$$

Proposition 5. If hypotheses $H(f)$ hold, then $\lambda_{*}>0$.

Proof. Arguing by contradiction, suppose that $\lambda_{*}=0$. Let $\left\{\lambda_{n}\right\}_{n \geqslant 1} \subseteq \mathcal{L}$ such that $\lambda_{n} \downarrow 0$ and let $u_{n} \in S_{\lambda_{n}} \subseteq \operatorname{int} C_{+}$for all $n \in \mathbb{N}$. We know that

$0 \leqslant u_{n} \leqslant \hat{u}_{\theta}$ for $\vartheta \geqslant \lambda_{0}$ big enough, for all $n \in \mathbb{N}$ (see the proof of Proposition 4),

$$
-\Delta u_{n}+u_{n}^{-\gamma}=\lambda_{n} f\left(z, u_{n}\right) \text { for almost all } z \in \Omega \text { and all } n \in \mathbb{N} \text {. }
$$


Let $\eta>0$. With $\rho=\left\|\hat{u}_{\theta}\right\|_{\infty}$ (see (26)), we have

$$
\begin{aligned}
-\Delta u_{n}+u_{n}^{-\gamma} & =\lambda_{n} f\left(z, u_{n}\right) \\
& \leqslant \lambda_{n} c_{\rho} u_{n}(\text { see }(20)) \\
& \leqslant \lambda_{n} c_{\rho} \hat{u}_{\theta}(\text { see }(26)) \\
& \leqslant \eta \hat{u}_{1} \text { for all } n \geqslant n_{0}\left(\text { recall that } \hat{u}_{1} \in \operatorname{int} C_{+}\right) .
\end{aligned}
$$

By (28) and Theorem 1(i) of Diaz, Morel \& Oswald [3] it follows that problem $(A u)_{\eta}$ has a positive solution. Since $\eta>0$ is arbitrary, we contradict Theorem 1(ii) of Diaz, Morel \& Oswald [3]. This proves that $\lambda_{*}>0$.

Proposition 6. If hypotheses $H(f)$ hold and $\lambda_{*}<\lambda$, then problem $\left(P_{\lambda}\right)$ has at least two positive solutions $u_{0}, \hat{u} \in \operatorname{int} C_{+}, u_{0} \neq \hat{u}$.

Proof. Let $\lambda_{*}<\sigma<\lambda<\mu$. On account of Proposition 4, we can find $u_{\sigma} \in S_{\sigma} \subseteq$ $\operatorname{int} C_{+}, u_{0} \in S_{\lambda} \subseteq \operatorname{int} C_{+}$and $u_{\mu} \in S_{\lambda} \subseteq \operatorname{int} C_{+}$such that

$$
\begin{aligned}
& u_{0}-u_{\sigma} \in \operatorname{int} C_{+} \text {and } u_{\mu}-u_{0} \in \operatorname{int} C_{+}, \\
\Rightarrow & u_{0} \in \operatorname{int}_{C_{0}^{1}(\bar{\Omega})}\left[u_{\sigma}, u_{\mu}\right] .
\end{aligned}
$$

We introduce the Carathéodory functions $e_{\lambda}(z, x)$ and $\hat{e}_{\lambda}(z, x)$ defined by

$$
\begin{gathered}
e_{\lambda}(z, x)= \begin{cases}\lambda f\left(z, u_{\sigma}(z)\right)-u_{\sigma}(z)^{-\gamma} & \text { if } x \leqslant u_{\sigma}(z) \\
\lambda f(z, x)-x^{-\gamma} & \text { if } u_{\sigma}(z)<x\end{cases} \\
\text { and } \hat{e}_{\lambda}(z, x)= \begin{cases}e_{\lambda}(z, x) & \text { if } x \leqslant u_{\mu}(z) \\
e_{\lambda}\left(z, u_{\mu}(z)\right) & \text { if } u_{\mu}(z)<x .\end{cases}
\end{gathered}
$$

We set $E_{\lambda}(z, x)=\int_{0}^{x} e_{\lambda}(z, s) d s$ and $\hat{E}_{\lambda}(z, x)=\int_{0}^{x} \hat{e}_{\lambda}(z, s) d s$ and consider the $C^{1}$-functionals $\beta_{\lambda}, \hat{\beta}_{\lambda}: H_{0}^{1}(\Omega) \rightarrow \mathbb{R}$ defined by

$$
\begin{aligned}
& \beta_{\lambda}(u)=\frac{1}{2}\|D u\|_{2}^{2}-\int_{\Omega} E_{\lambda}(z, u) d z, \\
& \hat{\beta}_{\lambda}(u)=\frac{1}{2}\|D u\|_{2}^{2}-\int_{\Omega} \hat{E}_{\lambda}(z, u) d z \text { for all } u \in H_{0}^{1}(\Omega) .
\end{aligned}
$$

Using (30), (31), as before (see the proof of Proposition 3), we can check that

$$
K_{\beta_{\lambda}} \subseteq\left[u_{\sigma}\right) \cap \operatorname{int} C_{+} \text {and } K_{\hat{\beta}_{\lambda}} \subseteq\left[u_{\sigma}, u_{\mu}\right] \cap \operatorname{int} C_{+} .
$$

Using (32), (30) and (29), we see that we may assume that

$$
K_{\beta_{\lambda}} \text { is finite and } K_{\beta_{\lambda}} \cap\left[u_{\sigma}, u_{\mu}\right]=\left\{u_{0}\right\} .
$$

Otherwise, we already have additional positive solutions and so we are done.

Evidently $\hat{\beta}_{\lambda}(\cdot)$ is coercive (see (30)). Also, it is sequentially weakly lower semicontinuous. Thus we can find $\hat{u}_{0} \in H_{0}^{1}(\Omega)$ such that

$$
\begin{aligned}
& \hat{\beta}_{\lambda}\left(\hat{u}_{0}\right)=\inf \left\{\hat{\beta}_{\lambda}(u): u \in H_{0}^{1}(\Omega)\right\}, \\
\Rightarrow \quad & \hat{u}_{0} \in K_{\hat{\beta}_{\lambda}} \subseteq\left[u_{\sigma}, u_{\mu}\right] \cap \operatorname{int} C_{+}(\operatorname{see}(32)) .
\end{aligned}
$$


From (30) and (31) we see that

$$
\begin{aligned}
& \left.\beta_{\lambda}^{\prime}\right|_{\left[u_{\sigma}, u_{\mu}\right]}=\left.\hat{\beta}_{\lambda}^{\prime}\right|_{\left[u_{\sigma}, u_{\mu}\right]}, \\
\Rightarrow & \hat{u}_{0} \in K_{\beta_{\lambda}} \cap\left[u_{\sigma}, u_{\mu}\right](\operatorname{see}(34)) \\
\Rightarrow & \hat{u}_{0}=u_{0}(\operatorname{see}(33)), \\
\Rightarrow & u_{0} \text { is a local } C_{0}^{1}(\bar{\Omega}) \text {-minimizer of } \beta_{\lambda}(\cdot), \\
\Rightarrow & \left.u_{0} \text { is a local } H_{0}^{1}(\Omega) \text {-minimizer of } \beta_{\lambda}(\cdot) \text { (see }[9]\right) \text {. }
\end{aligned}
$$

Then from (33) and Theorem 5.7.6 of Papageorgiou, Rădulescu \& Repovš [12, p. 367], we know that we can find $\rho \in(0,1)$ so small that

$$
\beta_{\lambda}\left(u_{0}\right)<\inf \left\{\beta_{\lambda}(u):\left\|u-u_{0}\right\|=\rho\right\}=m_{\lambda} .
$$

Hypothesis $H(f)(i i)$ implies that

$$
\beta_{\lambda}\left(t \hat{u}_{1}\right) \rightarrow-\infty \text { as } t \rightarrow+\infty \text {. }
$$

Finally, recall that hypothesis $H(f)($ iii $)$ implies that

$$
\beta_{\lambda}(\cdot) \text { satisfies the } \mathrm{C} \text {-condition }
$$

(see Papageorgiou \& Rădulescu [11]).

Then (35), (36), (37) permit the use of the mountain pass theorem. So, we can find $\hat{u} \in H_{0}^{1}(\Omega)$ such that

$$
\begin{aligned}
\hat{u} \in K_{\beta_{\lambda}} \text { and } m_{\lambda} \leqslant \beta_{\lambda}(\hat{u}), \\
\Rightarrow \quad \hat{u} \in S_{\lambda} \subseteq \operatorname{int} C_{+}, \hat{u} \neq u_{0}(\text { see }(32),(31) \text { and }(35)) .
\end{aligned}
$$

The proof is now complete.

Summarizing, we can state the following theorem for the set of positive solutions of problem $\left(P_{\lambda}\right)$.

Theorem 7. If hypotheses $H(f)$ hold, then there exists $\lambda_{*}>0$ such that

(a) for all $\lambda>\lambda_{*}$ problem $\left(P_{\lambda}\right)$ has at least two positive solutions

$$
u_{0}, \hat{u} \in \operatorname{int} C_{+}, u_{0} \neq \hat{u} \text {; }
$$

(b) for all $\lambda \in\left(0, \lambda_{*}\right)$ problem $\left(P_{\lambda}\right)$ has no positive solutions.

Remark 2. From the above Theorem is missing what happens at the critical case $\lambda=\lambda_{*}$. We were unable to resolve this case.

If $\lambda_{n} \downarrow \lambda_{*}$, then we can show that there exist $u_{n} \in S_{\lambda_{n}} \subseteq \operatorname{int} C_{+}(n \in \mathbb{N})$ such that

$$
u_{n} \stackrel{\mathrm{w}}{\rightarrow} u_{*} \text { in } H_{0}^{1}(\Omega), u_{*} \neq 0 .
$$

As before (see the proof of Proposition 3), we have

$$
u_{n}^{-\gamma} \in L^{s}(\Omega)(s>N) \text { and } u_{n}^{-\gamma} \rightarrow u_{*}^{-\gamma} \text { for almost all } z \in \Omega .
$$

However, we can not show that $\left\{u_{n}^{\gamma}\right\}_{n \geqslant 1} \subseteq L^{s}(\Omega)$ is bounded and therefore have that

$$
\int_{\Omega} u_{n}^{-\gamma} h d z \rightarrow \int_{\Omega} u_{*}^{-\gamma} h d z \text { for all } h \in H_{0}^{1}(\Omega)
$$

(Vitali's theorem, see Gasinski \& Papageorgiou [4, p. 901]).

In addition, we can not show that there exists $c_{*}>0$ such that

$$
u_{*} \geqslant c_{*} \hat{d} \text {. }
$$


It seems that $\lambda_{*}>0$ is not admissible (that is, $\lambda_{*} \notin \mathcal{L}$, hence $\mathcal{L}=\left(\lambda_{*},+\infty\right)$, see (25)), but this needs a proof.

Another open problem is the possibility of extending this work to equations driven by the $p$-Laplacian. This extension requires a corresponding generalization of the work of Diaz, Morel \& Oswald [3] to the case of the $p$-Laplacian. However, the tools of [3] are particular for the Laplacian. So, it is not clear how this generalization can be achieved. Hence new techniques are needed.

Acknowledgments. This research was supported by the Slovenian Research Agency grants P1-0292, J1-8131, J1-7025, N1-0064, and N1-0083, and the Romanian Ministry of Research and Innovation, CNCS-UEFISCDI, project number PN-III-P4-ID-PCE-2016-0130, within PNCDI III.

\section{REFERENCES}

[1] H. Brezis, Functional Analysis, Sobolev Spaces and Partial Differential Equations, Springer, New York, 2011.

[2] M. Coclite, G. Palmieri, On a singular nonlinear Dirichlet problem, Commun. Partial Differential Equations 14 (1989), 1315-1327.

[3] J.I. Diaz, J.M. Morel, L. Oswald, An elliptic equation with singular nonlinearity, Commun. Partial Differential Equations 12 (1987), 1333-1334.

[4] L. Gasinski, N.S. Papageorgiou, Nonlinear Analysis, Chapman \& Hall/CRC, Boca Raton, FL, 2016.

[5] M. Ghergu, V.D. Rădulescu, Singular Elliptic Problems: Bifurcation and Asymptotic Analysis, Clarendon Press, Oxford, 2008.

[6] D. Gilbarg, N.S. Trudinger, Elliptic Partial Differential Equations of Second Order (Second Edition), Springer, Berlin, 1998.

[7] Y. Haitao, Multiplicity and asymptotic behavior of positive solutions for a singular semilinear elliptic problem, J. Differential Equations 189 (2003), 487-512.

[8] A. Lazer, P.J. McKenna, On a singular nonlinear elliptic boundary value problem, Proc. Amer. Math. Soc. 111 (1991), 721-730.

[9] N.S. Papageorgiou, V.D. Rădulescu, Nonlinear nonhomogeneous Robin problems with superlinear reaction, Adv. Nonlin. Studies 16 (2016), 737-764.

[10] N.S. Papageorgiou, V.D. Rădulescu, Combined effects in some nonlinear elliptic problems, Nonlinear Anal. 109 (2014), 236-244.

[11] N.S. Papageorgiou, V.D. Rădulescu, Coercive and noncoercive nonlinear Neumann problems with indefinite potential, Forum Math. 28 (2016), 545-571.

[12] N.S. Papageorgiou, V.D. Rădulescu, D.D. Repovš, Modern Nonlinear Analysis-Theory, Springer Monographs in Mathematics, Springer, Cham, 2019 (ISBN 978-3-030-03429-0).

[13] Y. Sun, S. Wu, Y. Long, Combined effects of singular and superlinear nonlinearities in some singular boundary value problems, J. Differential Equations 176 (20010), 515-531.

[14] P. Tolksdorf, On the Dirichlet problem for quasilinear equations in domains with conical boundary points, Commun. Partial Differential Equations 8 (1983), 773-817.

(N.S. Papageorgiou) Institute of Mathematics, Physics and Mechanics, 1000 Ljubljana, Slovenia \& National Technical University, Zografou Campus, Athens 15780, Greece

E-mail address: npapg@math.ntua.gr

(V.D. Rădulescu) Institute of Mathematics, Physics and Mechanics, 1000 Ljubljana, Slovenia \& AGH University of Science and Technology, 30-059 Kraków, Poland

E-mail address: vicentiu.radulescu@imfm.si

(D.D. Repovš) Faculty of Education and Faculty of Mathematics and Physics, University of Ljubljana \& Institute of Mathematics, Physics and Mechanics, 1000 Ljubljana, SLOVENIA

E-mail address: dusan.repovs@guest.arnes.si 